\section{Bringt eine sequenzielle Therapie Vorteile?}

\author{
Eine Nagelmykose erfolgreich zu therapieren ist schwierig. \\ Gerade bei Monotherapien sind die Heilungschancen relativ \\ gering. Die Wissenschaftler um C. Paul haben in einer Studie \\ die Wirksamkeit einer sequenziellen Therapie (SEQ) analysiert. \\ Dermatology 2013; 227: 157-164
}

Die Onychomykose ist ein verbreitetes Problem, obwohl zahlreiche lokale und systemische antifungale Wirkstoffe zur Verfügung stehen. In einer großen europäischen Studie namens „Achilles" waren knapp 41 \% der Teilnehmer von einer Fußpilzerkrankung betroffen. Die Onychomykose kam dabei am häufigsten vor. Die Wissenschaftler haben nun die Wirksamkeit einer SEQ, mit der alleinigen Anwendung eines Amorolfin-haltigen Nagellack verglichen.

An der Studie nahmen 17 Zentren in den Jahren 2009-2011 in Frankreich und Tunesien teil. Die Patienten wurden auf 2 Gruppen randomisiert: Eine Gruppe erhielt die ureahaltige Salbe zur Nagelablösung, kombiniert mit einer SEQ-Therapie mit $1 \%$ iger Ciclopiroxolamin-Creme und einer $8 \%$ iger Ciclopirox-Lösung. Die ureahaltige Salbe wurde 1-mal täglich aufgetragen und für 24 Stunden mit einem speziellen Pflaster abgeklebt. Diese Therapie wurde 3 Wochen lang durchgeführt. Dann erfolgte ein Débridement sowie eine 8-wöchige Therapie mit 1\% iger Ciclopiroxolamin-Creme. Danach wendeten die Patienten 2-mal pro Woche einen $8 \%$ igen Ciclopirox-Nagellack über 25 Wochen an. Die Kontrollgruppe wurde mit AmorolfinHydrochlorid 2-mal pro Woche über 36 Wochen behandelt. Die Studie dauerte insgesamt 48 Wochen.

\section{Sequenzbehandlung wirksamer als Monotherapie $\nabla$}

An der Studie nahmen 142 Patienten teil: je 71 erhielten die Amorolfin- bzw. die SEQ-Therapie. 95,1\% der Patienten komplettierten die Studie. 5 Patienten (7\%) der Amorolfin-Gruppe und 2 Patienten $(2,8 \%)$ der SEQ-Gruppe beendeten die

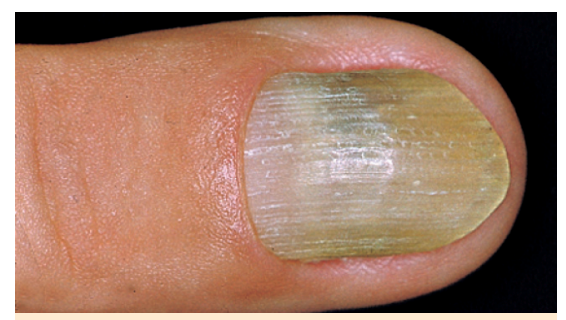

Eine Monotherapie ist bei Nagelmykose oft

Teilnahme vorzeitig. Die Compliance betrug 94,6\% in der Amorolfin-Gruppe und $95,2 \%$ in der SEQ-Gruppe.

Nach 48 Wochen waren 36,6\% der Patienten der SEQ-Gruppe vollständig geheilt, aber nur 12,7\% der Amorolfin-Gruppe $(\mathrm{p}=0,001, \mathrm{OR}=3,98 ; 95 \%$-Konfidenzintervall $[\mathrm{KI}]:$ 1,70-9,30). Als klinisch geheilt galten 53,5\% der SEQ- und 16,9\% der Amorolfin-Gruppe ( $p=0,001, \quad O R=5,66$; 95\%-KI 2,6-12,31). Die sequenzielle Behandlung wurde besser vertragen als die Amorolfin-Behandlung: Während bei 21 Patienten (30\%) der Amorolfin-Gruppe mind. eine unerwünschte Wirkung auftrat, war dies nur bei 13 Patienten $(18,1 \%)$ der SEQ-Gruppe der Fall. Die Kosten für die erfolgreiche Behandlung eines Patienten betrugen $76 €$ bei der Amorolfin-Behandlung und $33 €$ bei der sequenziellen Therapie.

\section{Fazit}

Eine Monotherapie reicht bei Onychomykose häufig nicht aus. Eine sequenzielle Therapie mit ureahaltiger Salbe, Ciclopiroxolamin-Creme und CiclopiroxNagellack führt im Gegensatz zu einer alleinigen Behandlung mit Amorolfin zu deutlich höheren Erfolgsraten und niedrigeren Kosten.

\section{Dr. Dunja Voos, Pulheim} nicht ausreichend (Bild: Thieme).
Allergologie

\section{Genexpression in Mastzellen kartiert}

Den Wissenschaftlern um E. Motakis ist es gelungen, das vollständige Transkriptom von menschlichen Immunzellen aufzuklären. Die Ergebnisse wurden im März in den Fachzeitschriften Blood und Nature veröffentlicht. Im Rahmen des internationalen Fantoms5-Konsortiums (Functional Annotation of the Mammalian Genome) haben die Wissenschaftler eine Art Atlas erstellt, der für nahezu jedes Gewebe dokumentiert, welche spezifischen Gene von welchem Zelltyp abgelesen werden. Die Wissenschaftler der Charité fokussierten ihren Teil des Projekts auf die Mastzellen. Diese setzen bei Aktivierung verschiedene Botenstoffe, u.a. Histamin, frei, was zu Allergien und Juckreiz führen kann.

\section{Die Wandlungsfähigkeit der Mastzellen \\ $\nabla$}

Durch eine spezielle Technik Cap Analysis of Gene Expression, CAGE) gelang es, jene Steuerungselemente im Genom der Mastzellen zu lokalisieren, die für das Ablesen der Erbinformationen in den Zellen zuständig sind. Des Weiteren konnten sie zeigen, dass Mastzellen auch Gene aktivieren, die normalerweise in anderen Zelltypen vorkommen. Sie fanden heraus, dass sich Mastzellen abhängig von der Umgebung, in die sie eingebettet sind, stark verändern. Dies unterstreicht ihre große Wandlungsfähigkeit.

„Der besondere Clou unserer Arbeit liegt darin, dass die Mastzellen nicht isoliert betrachtet wurden, sondern in das Fantoms5-Projekt eingebettet waren, welches rund 1000 Proben parallel untersucht hat“, so die Forscher. „Dadurch ließ sich die Genexpression in Mastzellen unmittelbar ins Verhältnis zu nahezu allen Geweben und Zellen des menschlichen Körpers setzen, wodurch eine quantitative Einordnung erfolgen konnte“. Denn erst wenn die Funktionalitäten von Mastzellen besser verstanden sind, lassen sich auch gezielt neue Therapieansätze für allergische und andere Erkrankungen etablieren.

Nach einer Mitteilung der Charité Berlin 